\title{
TORINO PROGRAMME ON RADIOSOURCES ASTROMETRY: PROGRESS REPORT.
}

\author{
G. Chiumiento ${ }^{(1)}$, M.G. Lattanzi ${ }^{(1,2)}$, G. Massone ${ }^{(1)}$,

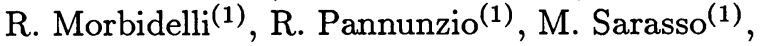 \\ (1) Torino Astronomical Observatory, 10025 Pino Torinese (TO), Italy \\ (2) Space Telescope Science Institute, Baltimore MD 21218, USA
}

\begin{abstract}
Measurements of precise positions of optical counterparts of extragalactic radio-sources, with the aim to compare them with VLBI positions, is in progress at Torino Observatory. The Carlsberg Automatic Meridian Circle Catalogue (CAMC) is used for plate reductions. Presently, about $40 \%$ of the plate material required to complete the project has been taken and some significant improvements have been made both in the material and the reduction methods. Improvements are also due to the publication of a new volume of the CAMC catalogue that significantly increases the number of stars in the fields.
\end{abstract}

\section{INTRODUCTION}

In a previous paper, (Chiumiento et al, 1989, hereinafter paper I) we reported some preliminary results on a programme of determination of precise optical positions of extragalactic radio-sources. Our observing list is a sample of 75 targets from the list proposed by the Working Group of the IAU Commission no. 24 (Argue et al, 1984). The selection criteria and a description of the telescopes used are described in paper I. The primary reference stars have been taken from the Carlsberg Meridian Catalogues no. 1, 2, 3 (La Palma: 1985, 1986, 1987).

During that first attempt, in spite of the small number of plates available, we obtained fairly good results. At this time, the plate material has been increased considerably and about $40 \%$ of the programme completed. Additionally, the following improvements have been introduced as standard procedure: a) 3 plates are taken for each object both at the $38 \mathrm{~cm}$ photographic refractor and the $105 \mathrm{~cm}$ astrometric reflector; b) the plates have been measured in direct and reverse directions with the digitized Ascorecord machine of Torino Astronomical Observatory; c) due to the increase in the number of CAMC stars, more sophisticated plate models than the six-constant linear one may be attempted. Second order effects, coma and magnitude terms can also be checked for; d) the secondary reference frame stars were increased from 10 to 15 . They were selected within $12^{\prime}$ the radiosource. The magnitude of these stars is intermediate between that of the CAMC stars and the radiosources.

\section{REDUCTION AND EVALUATION OF INTERNAL ERROR}

The reduction procedure of our material (internal error evaluation and quality 501

J. H. Lieske and V. K. Abalakin (eds.), Inertial Coordinate System on the Sky, 501-502. (c) 1990 IAU. Printed in the Netherlands. 
control) may be summarized as follows: 1) measure the refractor plates in direct and reverse directions; 2 ) combine the two sets for the same plate and evaluate the inter-agreement of the measurements $\sigma_{m}$ (column 1 in Table 1);3) derive RA and DEC for the intermediate reference stars; their internal error is measured through the mean error of unit weight $\sigma_{0}$ of the adjustment with the CAMC stars (column 2); this must be consistent with the error obtained at step 2 plus the error of CAMC positions; 4) average the individual positions of the intermediate stars from different refractor plates; the dispersion of these positions is reported in column 3 of Table $1 ; 5$ ) repeat steps 1 to 4 for reflector plates using the intermediate stars as reference frame and the target radiosources as test stars.

Column 4 in Table 1 was computed using the formula $\sigma_{m}^{2}+\sigma_{0}^{2} / n$ where $n$ is the number of reference stars. The figures in columns 3 and 4 would match. However, we can see that the match is satisfactory for refractor plates only. The poor result for reflector plates seems to be due to the low quality of faintest radiosources images. This can be inferred, on a plate basis, by comparing the value $0^{\prime \prime} .076$ in column 1 of Table 1 with the corresponding $0^{\prime \prime} .053$ obtained for the secondary reference stars images on the reflector plates. This value is very close to what expected considering the more favourable scale $(\mathrm{S})$ of the reflector $\left(S_{\text {reflector }} / S_{\text {refractor }} \simeq 1.43\right)$. That image quality in our measurements is strongly dependent on the magnitude of the radiosources, is clearly confirmed in Figure 1. Here, the individual values used in computing the average in column 3 of Table 1 are given.

Comparison of our preliminary optical positions with the radio ones in the catalogue by Argue et al. gave the following mean residuals:

$$
<\Delta \alpha>\simeq 0^{\prime \prime} .18<\Delta \delta>\simeq 0^{\prime \prime} .15
$$

Seeking for improvements on the faintest target positions, some of the plates will be re-measured with a PDS. If necessary, new plates will be taken for the worst cases.

Table 1. Internal error table

\begin{tabular}{cccc}
\hline \multicolumn{4}{c}{ Refractor plates } \\
1 & 2 & 3 & 4 \\
$0^{\prime \prime} .082$ & $0^{\prime \prime} .185$ & $0^{\prime \prime} .117$ & $0^{\prime \prime} .086$ \\
\multicolumn{4}{c}{ Reflector plates } \\
1 & 2 & 3 & 4 \\
$0^{\prime \prime} .076$ & $0^{\prime \prime} .094$ & $0^{\prime \prime} .233$ & $0^{\prime \prime} .079$ \\
& & &
\end{tabular}

\section{REFERENCES}

Argue, A. et al.: 1984, Astron. Astrophys. 130, 191.

Chiumiento et al.: IAU Coll. No. 100, Belgrade 1987, Cel. Mechanics, in press. 